\title{
Experiments on the Automated Selection of Patients for Clinical Trials
}

\author{
Eugene Fink \\ eugene@csee.usf.edu \\ Bhavesh D. Goswami \\ bgoswami@csee.usf.edu
}

\author{
Lawrence O. Hall \\ hall@csee.usf.edu \\ Matthew Boonstra \\ boonstra@csee.usf.edu
}

\author{
Dmitry B. Goldgof \\ goldgof@csee.usf.edu
}

\author{
Jeffrey P. Krischer \\ jpkrischer@moffitt.usf.edu
}

Computer Science and Engineering, University of South Florida, Tampa, Florida 33620

\begin{abstract}
When clinicians test a new treatment procedure, they need to identify and recruit patients with appropriate medical conditions. We have developed an expert system that helps clinicians select patients for experimental treatments, and to reduce the number and overall cost of related medical tests. We describe experiments on selecting patients for new treatments at the Moffitt Cancer Center. The experiments have shown that the system can increase the number of selected patients by a factor of three, and that it can also reduce the cost of the selection process.
\end{abstract}

Keywords: Medical expert systems, breast cancer, cost reduction.

\section{Introduction}

When clinicians conduct treatment experiments, called clinical trials, they have to recruit participants from current patients. To select prospective participants, clinicians analyze the data of available patients and identify patients with appropriate medical conditions. This analysis has traditionally been a manual process, and studies have shown that clinicians miss up to $60 \%$ of the matching patients, which delays the completion of clinical trials $[7,17]$.

To address this problem, several researchers built expert systems to help clinicians select trial participants. Ohno-Machado et al. developed the AIDS $^{2}$ system, which selected AIDS patients for clinical trials [11]. Musen et al. built a rule-based system, called EON, that also selected AIDS trial participants [8]. Bouaud et al. created the ONCODOC system, which suggested trials for cancer patients $[2,3]$. Séroussi et al. used ONCODOC to identify trial participants at two hospitals, which helped in-

0-7803-7952-7/03/\$17.00 (C) 2003 IEEE. crease the number of selected patients by a factor of three $[13,14,15]$.

The National Cancer Institute created a search engine for selecting clinical trials, available through the Internet at www.cancer.gov/search/clinical_trials. It prompts a user to answer several questions about a patient, and gives a list of potentially matching trials; however, it does not determine whether the patient satisfies all of the requirements of these trials.

Fallowfield et al. studied how physicians selected cancer patients for clinical trials, and compared manual and automated selection [5]. They showed that expert systems could improve the selection accuracy, but physicians were reluctant to use these systems. Carlson et al. conducted similar studies with AIDS trials, and also concluded that expert systems could lead to a more accurate selection [4].

A recent project at the University of South Florida has also been aimed at automated identification of prospective trial participants. Theocharous developed a Bayesian system that selected clinical trials for cancer patients $[12,16]$, and Bhanja et al. built a qualitative rule-based system for the same task [1].

We have continued their work, built a new version of the rule-based system $[6,9,10]$, and applied it to selecting patients for breast-cancer trials at the Moffitt Cancer Center, located on campus of the University of South Florida. We outline the design of this system and present an empirical evaluation of its effectiveness.

\section{Knowledge base}

Physicians at the Moffitt Cancer Center currently conduct about 150 clinical trials. We have developed an expert system to help physicians select trials for eligible patients; it consists of a knowledge base and a webbased interface for entering patient data. The knowledge base contains information about related medical 
(a) MEDICAL TESTS

General information

What is the patient's sex?

What is the patient's age?

Mammogram, Cost is $\$ 150$

What is the cancer stage?

Does the patient have invasive cancer?

Biopsy, Cost is $\$ 400$

How many lymph nodes have tumor cells?

What is the greatest tumor diameter?

Electrocardiogram, Cost is $\$ 200$

Does the patient have cardiac arrhythmias?

(b) ELIGIBILITY CRITERIA

sex $=$ FEMALE and

age $\leq 45$ and

cancer-stage $\in\{\mathrm{II}, \mathrm{III}\}$ and

invasive-cancer $=\mathrm{NO}$ and

lymph-nodes $\leq 3$ and

(arrhythmias $=$ NO or

tumor-diameter $\leq 2.5)$

Figure 1: Description of medical tests and trialeligibility criteria in the trial-selection system.

tests, as well as logical expressions that determine a patient's eligibility for each trial. The description of a medical test includes its dollar cost and list of questions that can be answered based on the test results (Figure 1a). The trial-eligibility criteria are represented by a logical expression, which includes variables that represent the patient data, as well as equalities, inequalities, "set-element" relations, conjunctions, and disjunctions (Figure 1b).

The system collects data until it can determine whether the eligibility expression is TRUE or FALSE. For example, if a clinician uses the system to determine a patient's eligibility for the trial in Figure 1(b), it first asks about the patient's sex and age. If the patient satisfies the corresponding conditions, it asks for the mammogram results, and then requests the biopsy and electrocardiogram data. The ordering of tests depends on their costs and on the amount of information provided by test results. The system begins with the mammogram because it is cheaper than the other tests and provides data for two clauses of the eligibility expression.

\section{$3 \quad$ Selection of participants}

We have built a knowledge base for the breast-cancer trials at the Moffitt Cancer Center, including five completed trials and ten current trials, and applied the system to retrospective data from the Moffitt patients who have had a breast-cancer surgery in the last three years. We have discarded the patients whose available records are incomplete, and used all remaining patients, which include 187 past patients and 169 current patients.

We have compared the results of automated trial selection for these patients with the manual selection by Moffitt clinicians. The system has identified all eligible patients for each trial, whereas the clinicians have selected about one-third of the eligible patients. We summarize the results for the past patients in Table 1(a), and the results for the current patients in Table 1(b). The "participants" column shows the number of actual participants of each trial; the "other eligible" column gives the number of the other eligible patients identified by the system.

For every current patient who did not participate in a matching trial, we have checked whether she participated in any other trial, and we show the results in Table 2. We have not done a similar analysis for the past patients due to insufficient data. The "incompatible" column in Table 2 includes the number of eligible patients who did not participate in a specified trial because of participation in another incompatible trial. The "compatible" column shows the number of patients who participated in another compatible trial, and could also have participated in the specified trial. Finally, the "no other trial" column gives the number of eligible patients who have not participated in any trial.

The results show that the system can identify eligible patients who have not been selected by clinicians; thus, it can increase the number of trial participants. For the patients in the reported experiments, it could increase the overall number of participants by a factor of three. In particular, it has found prospective participants for some trials with a very small number of manually selected patients. For example, it has found nineteen matching patients for trial 12385 , which currently has no participants, and twenty-six patients for trial 11931, which has only two participants.

\section{Cost reduction}

If the available patient records do not provide enough data for trial selection, clinicians perform medical tests as part of the selection process. They can reduce the overall test cost by first ordering inexpensive tests, and then using their results to avoid some expensive tests.

The system suggests the ordering of tests that reduces their expected cost. After getting the results of the first test, it re-evaluates the need for the other tests and revises their ordering. The choice of the first test is based on three criteria. The system scores all required tests according to these criteria, computes a linear combination of the three scores for every test, and chooses the test with the highest score. 
Table 1: Results of selecting clinical trials for the 187 past patients and 169 current patients. We give the number of trial participants, selected by both the system and Moffitt clinicians, and the number of the other eligible patients, identified by the system.

(a) Results for the 187 past patients.

\begin{tabular}{|c|c|c|}
\hline $\begin{array}{c}\text { Clinical } \\
\text { Trial }\end{array}$ & $\begin{array}{c}\text { Parti- } \\
\text { cipants }\end{array}$ & $\begin{array}{c}\text { Other } \\
\text { Eligible }\end{array}$ \\
\hline 10822 & 10 & 5 \\
10840 & 0 & 19 \\
11072 & 48 & 26 \\
11378 & 4 & 19 \\
11992 & 5 & 6 \\
12100 & 8 & 20 \\
12101 & 20 & 30 \\
\hline
\end{tabular}

(b) Results for the 169 current patients.

\begin{tabular}{|c|c|c|}
\hline $\begin{array}{c}\text { Clinical } \\
\text { Trial }\end{array}$ & $\begin{array}{c}\text { Parti- } \\
\text { cipants }\end{array}$ & $\begin{array}{c}\text { Other } \\
\text { Eligible }\end{array}$ \\
\hline 11132 & 4 & 1 \\
11931 & 2 & 26 \\
11971 & 4 & 0 \\
12100 & 0 & 5 \\
12101 & 11 & 52 \\
12385 & 0 & 19 \\
12601 & 0 & 1 \\
12643 & 16 & 36 \\
12757 & 1 & 3 \\
12775 & 23 & 17 \\
\hline
\end{tabular}

Table 2: Participation of the patients who skipped a matching clinical trial in other trials. We show the number of patients who skipped the trial because of participation in another incompatible trial; the number of patients who were on another trial compatible with the skipped trial; and the number of eligible patients who were not on any trial.

\begin{tabular}{|c|c|c|c|}
\hline $\begin{array}{c}\text { Clinical } \\
\text { Trial }\end{array}$ & $\begin{array}{c}\text { Incom- } \\
\text { patible }\end{array}$ & $\begin{array}{c}\text { Compa- } \\
\text { tible }\end{array}$ & $\begin{array}{c}\text { No Other } \\
\text { Trial }\end{array}$ \\
\hline 11132 & 0 & 1 & 0 \\
11931 & 0 & 11 & 15 \\
11971 & 0 & 0 & 0 \\
12100 & 0 & 1 & 4 \\
12101 & 13 & 6 & 33 \\
12385 & 8 & 2 & 9 \\
12601 & 0 & 0 & 1 \\
12643 & 0 & 10 & 26 \\
12757 & 0 & 1 & 2 \\
12775 & 3 & 3 & 11 \\
\hline
\end{tabular}

Table 3: Cost savings by test reordering.

(a) Results for the 187 past patients.

\begin{tabular}{|c|c|c|}
\hline \multirow{2}{*}{$\begin{array}{c}\text { Clinical } \\
\text { Trial }\end{array}$} & \multicolumn{2}{|c|}{ Mean Cost } \\
\cline { 2 - 3 } & Weordering & $\begin{array}{c}\text { With Test } \\
\text { Reordering }\end{array}$ \\
\hline 10822 & $\$ 70$ & $\$ 11$ \\
10840 & $\$ 0$ & $\$ 0$ \\
11072 & $\$ 209$ & $\$ 60$ \\
11378 & $\$ 35$ & $\$ 19$ \\
11992 & $\$ 0$ & $\$ 0$ \\
12100 & $\$ 0$ & $\$ 0$ \\
12101 & $\$ 0$ & $\$ 0$ \\
\hline
\end{tabular}

(b) Results for the 169 current patients.

\begin{tabular}{|c|c|c|}
\hline \multirow{2}{*}{ Clinical } & \multicolumn{2}{|c|}{ Mean Cost } \\
\cline { 2 - 3 } Trial & W/O Test & With Test \\
& Reordering & Reordering \\
\hline 11132 & $\$ 0$ & $\$ 0$ \\
11931 & $\$ 0$ & $\$ 0$ \\
11971 & $\$ 192$ & $\$ 192$ \\
12100 & $\$ 0$ & $\$ 0$ \\
12101 & $\$ 0$ & $\$ 0$ \\
12385 & $\$ 0$ & $\$ 0$ \\
12601 & $\$ 36$ & $\$ 3$ \\
12643 & $\$ 0$ & $\$ 0$ \\
12757 & $\$ 107$ & $\$ 107$ \\
12775 & $\$ 0$ & $\$ 0$ \\
\hline
\end{tabular}

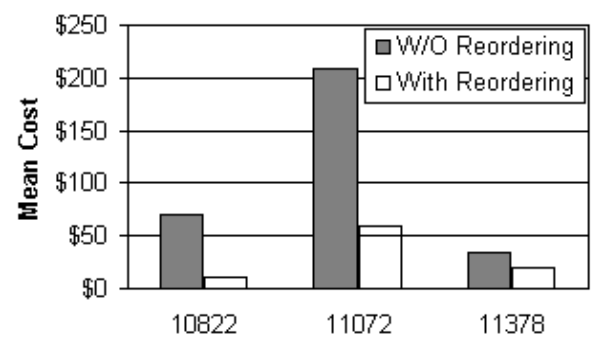

(a) Results for the 187 past patients.

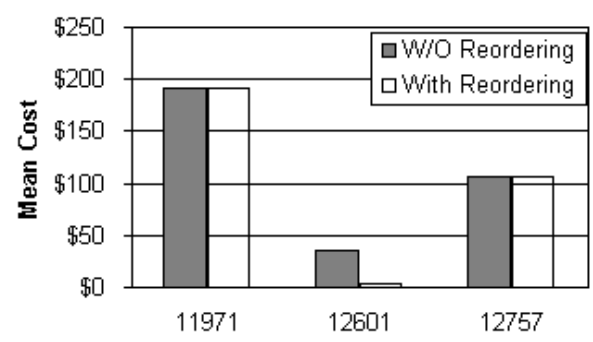

(b) Results for the 169 current patients.

Figure 2: Costs with and without test reordering. We plot the results for the six clinical trials that have incurred nonzero selection costs. 
1. Cost of a test. The system gives preference to less expensive tests.

2. Immediate decision. If a test can lead to an immediate acceptance or rejection of the trial, the system prefers it to other tests.

3. Number of related clauses. The system prefers the tests that provide data for large number of clauses in the eligibility expression.

The system disregards the costs of tests performed in the normal course of treatment, and accounts only for the costs related to the trial selection. For example, if a patient needs a mammogram regardless of trial participation, the system views it as a zero-cost test. On the other hand, if the only purpose of the biopsy and electrocardiogram is to select trials, the system uses the heuristics to order these tests.

We show the mean test costs with and without the ordering heuristics in Table 3, and give a graphical view of the cost savings in Figure 2. The results confirm that the heuristics reduce the cost of the selection process. Six clinical trials have incurred selection costs; the heuristics have reduced the costs for four of these trials, and have not affected the costs for the other two trials.

The results in Table 3(a) differ from similar experiments with an earlier version of the system [6], because of two changes to the system. First, the current version disregards the costs of the tests required for the regular treatment, which do not affect the trial-selection expenses, whereas the earlier version counted all costs. Second, some costs in the old system were out-of-date, and we have corrected them based on the data from the Moffitt accounting department.

\section{Reduction of data entry}

The system tries to minimize not only the overall cost of medical tests, but also the amount of data entry, that is, the number of questions asked about a patient. For each question, it estimates the probability that the answer will lead to an immediate acceptance or rejection of the trial, and it gives preference to the questions with the highest probability of an immediate decision. Thus, when a clinician enters the available data, the system asks the related questions in the decreasing order of the immediate-decision probabilities. It estimates these probabilities from past experience with other patients. For each question, it determines the percentage of past answers that have led to immediate decisions, and uses this percentage as the probability estimate.

We have evaluated the effectiveness of this ordering heuristic for six clinical trials, using the data from the 169 current patients. We have performed ten-fold crossvalidation; that is, we have used $90 \%$ of the patients to compute the related probabilities, and then measured the mean number of questions for the other $10 \%$.

We show the results with and without the ordering heuristic in Table 4, and give a graphical view of the
Table 4: Reduction of data entry by the reordering of questions, for the 169 current patients.

\begin{tabular}{|c|c|c|}
\hline \multirow{2}{*}{$\begin{array}{c}\text { Clinical } \\
\text { Trial }\end{array}$} & \multicolumn{2}{|c|}{ Mean Number of Questions } \\
\cline { 2 - 3 } & Reordering & $\begin{array}{c}\text { With Question } \\
\text { Reordering }\end{array}$ \\
\hline 11931 & 18.9 & 15.4 \\
12100 & 14.0 & 13.9 \\
12101 & 24.8 & 21.7 \\
12385 & 19.1 & 14.8 \\
12601 & 15.7 & 13.9 \\
12775 & 16.1 & 14.4 \\
\hline
\end{tabular}

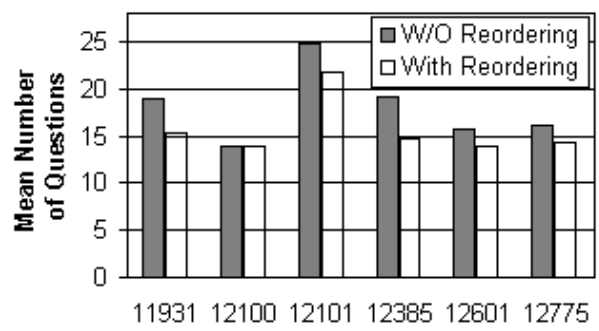

Figure 3: Number of questions with and without the reordering heuristic, for the 169 current patients.

same results in Figure 3. The heuristic has reduced the number of questions for all six trials; the reduction ranges from $1 \%$ to $29 \%$, and its mean is $15 \%$. The results confirm that the accumulated statistical data help reduce the number of questions.

\section{Concluding remarks}

We have developed an expert system that selects clinical trials for eligible patients. Experiments have confirmed that the system can increase the number of clinical-trial participants. They have also shown that the ordering of related medical tests affects the overall test cost, and the implemented heuristics can reduce this cost.

Acknowledgments: This work has been partially supported by the Moffitt Cancer Center and by the Breast Cancer Research Program of the U.S. Army Medical Research and Materiel Command under contract DAMD1700-1-0244.

\section{References}

[1] Sanjukta Bhanja, Lynn M. Fletcher, Lawrence O. Hall, Dmitry B. Goldgof, and Jeffrey P. Krischer. A qualitative expert system for clinical trial assignment. In Proceedings of the Eleventh International Florida Artificial Intelligence Research Society Conference, pages 84-88, 1998.

[2] Jacques Bouaud, Briggite Séroussi, Éric-Charles Antoine, Mary Gozy, David Khayat, and Jean- 
Franşois Boisvieux. Hypertextual navigation operationalizing generic clinical practice guidelines for patient-specific therapeutic decisions. Journal of the American Medical Informatics Association, 5(suppl.):488-492, 1998.

[3] Jacques Bouaud, Briggite Séroussi, Éric-Charles Antoine, Laurent Zelek, and Marc Spielmann. Reusing ONCODOC, a guideline-based decision support system, across institutions: A successful experiment in sharing medical knowledge. In Proceedings of the American Medical Informatics Association Annual Symposium, volume 7, 2000.

[4] Robert W. Carlson, Samson W. Tu, Nancy M. Lane, Tze L. Lai, Carol A. Kemper, Mark A. Musen, and Edward H. Shortliffe. Computer-based screening of patients with HIV / AIDS for clinical trial eligibility. Online Journal of Current Clinical Trials, 4(179), 1995.

[5] Lesley Fallowfield, D. Ratcliffe, and Robert Souhami. Clinicians' attitudes to clinical trials of cancer therapy. European Journal of Cancer, 33(13):2221-2229, 1997.

[6] Princeton K. Kokku, Lawrence O. Hall, Dmitry B. Goldgof, Eugene Fink, and Jeffrey P. Krischer. A cost-effective agent for clinical trial assignment. In Proceedings of the IEEE International Conference on Systems, Man, and Cybernetics, 2002.

[7] Cyrus Kotwall, Leo J. Mahoney, Robert E. Myers, and Linda Decoste. Reasons for non-entry in randomized clinical trials for breast cancer: A single institutional study. Journal of Surgical Oncology, 50:125-129, 1992.

[8] Mark A. Musen, Samson W. Tu, Amar K. Das, and Yuval Shahar. EON: A component-based approach to automation of protocol-directed therapy. Journal of the American Medical Informatics Association, 3(6):367-388, 1996.

[9] Savvas Nikiforou. Selection of clinical trials: Knowledge representation and acquisition. Master's thesis, Department of Computer Science and Engineering, University of South Florida, 2002.

[10] Savvas Nikiforou, Eugene Fink, Lawrence O. Hall, Dmitry B. Goldgof, and Jeffrey P. Krischer. Knowledge acquisition for clinical-trial selection. In Proceedings of the IEEE International Conference on Systems, Man, and Cybernetics, 2002.

[11] Lucila Ohno-Machado, Eduardo Parra, Suzanne B. Henry, Samson W. Tu, and Mark A. Musen. AIDS ${ }^{2}$ : A decision-support tool for decreasing physicians' uncertainty regarding patient eligibility for HIV treatment protocols. In Proceedings of the Seventeenth Annual Symposium on Computer Applications in Medical Care, pages 429-433, 1993.

[12] Constantinos Papaconstantinou, Georgios Theocharous, and Sridhar Mahadevan. An expert system for assigning patients into clinical trials based on Bayesian networks. Journal of Medical Systems, 22(3):189-202, 1998.

[13] Briggite Séroussi, Jacques Bouaud, and ÉricCharles Antoine. Users' evaluation of ONCODOC, a breast cancer therapeutic guideline delivered at the point of care. Journal of the American Medical Informatics Association, 6(5):384-389, 1999.

[14] Briggite Séroussi, Jacques Bouaud, and ÉricCharles Antoine. ONCODOC: A successful experiment of computer-supported guideline development and implementation in the treatment of breast cancer. Artificial Intelligence in Medicine, 22(1):43-64, 2001.

[15] Briggite Séroussi, Jacques Bouaud, Éric-Charles Antoine, Laurent Zelek, and Marc Spielmann. Using ONCODOC as a computer-based eligibility screening system to improve accrual onto breast cancer clinical trials. In Silvana Quaglini, Pedro Barahona, and Steen Andreassen, editors, Artificial Intelligence in Medicine, pages 421-430. SpringerVerlag, Berlin, Germany, 2001.

[16] Georgios Theocharous. An expert system for assigning patients into clinical trials based on Bayesian networks. Master's thesis, Computer Science and Engineering Department, University of South Florida, 1996.

[17] Samson W. Tu, Carol A. Kemper, Nancy M. Lane, Robert W. Carlson, and Mark A. Musen. A methodology for determining patients' eligibility for clinical trials. Journal of Methods of Information in Medicine, 32(4):317-325, 1993. 